\title{
Impact of adolescent ethanol exposure and adult amphetamine self-administration on evoked striatal dopamine release in male rats
}

\author{
L. Granholm $^{1}$ • S. Rowley ${ }^{1}$ - M. Ellgren ${ }^{1}$ - L. Segerström ${ }^{1}$ - I. Nylander ${ }^{1}$
}

Received: 19 February 2015 / Accepted: 31 August 2015 / Published online: 26 September 2015

(C) The Author(s) 2015. This article is published with open access at Springerlink.com

\begin{abstract}
Rationale Adolescent binge drinking is common and associated with increased risk of substance use disorders. Transition from recreational to habitual ethanol consumption involves alterations in dorsal striatal function, but the long-term impact of adolescent ethanol exposure upon this region remains unclear.

Objectives This study aimed to characterise and describe relationships between adolescent ethanol exposure, amphetamine self-administration and adult dopamine dynamics in dorsal striatum, including response to amphetamine challenge, in male Wistar rats.

Methods Ethanol $(2 \mathrm{~g} / \mathrm{kg})$ or water was administered intragastrically in an episodic binge-like regimen (three continuous days/week) between 4 and 9 weeks of age (i.e. postnatal days 28-59). In adulthood, animals were divided into two groups. In the first, dorsal striatal potassium-evoked dopamine release was examined via chronoamperometry, in the basal state and after a single amphetamine challenge $(2 \mathrm{mg} / \mathrm{kg}$, i.v.). In the second, amphetamine self-administration behaviour was studied (i.e. fixed and progressive ratio) before chronoamperometric analysis was conducted as described above.

Results Adolescent ethanol exposure suppressed locally evoked dopamine response after amphetamine challenge in adulthood, whereas in the basal state, no differences in
\end{abstract}

L. Granholm and S. Rowley contributed equally to this work.

L. Granholm

linnea.granholm@farmbio.uu.se

1 Department of Pharmaceutical Biosciences,

Division of Neuropharmacology, Addiction and Behaviour,

Uppsala University, Uppsala, Sweden dopamine dynamics were detected. Ethanol-exposed animals showed no differences in adult amphetamine selfadministration behaviour but an abolished effect on dopamine removal in response to a single amphetamine challenge after self-administration.

Conclusion Amphetamine challenges in adult rats revealed differences in in vivo dopamine function after adolescent ethanol exposure. The attenuated drug response in ethanolexposed animals may affect habit formation and contribute to increased risk for substance use disorders as a consequence of adolescent ethanol.

Keywords Chronoamperometry $\cdot$ Operant self-administration $\cdot$ Alcohol $\cdot$ Rodents
Abbreviations
FR Fixed ratio
PR Progressive ratio
PND Post-natal day

\section{Introduction}

Adolescence represents a period of extensive reorganisation and maturation of brain circuits involved in emotions, motivation and cognition. This age is associated with high impulsivity, reduced behavioural control (Adriani and Laviola 2003; Arnett 1992; for review, see Spear 2000; Steinberg 2008) and altered risk valuation and decision making (Nasrallah et al. 2011; Nasrallah et al. 2009; Steinberg et al. 2009). These traits are commonly linked to increased risk for excessive drug consumption.

Alcohol is widely used among young people, and the consumption of the drug increases throughout adolescence 
(Swendsen et al. 2012). The age of first alcohol consumption is associated with both adult risk for alcohol use disorder (Dawson et al. 2008; DeWit et al. 2000; Grant and Dawson 1997; Hawkins et al. 1997) and substance use disorders (Anthony and Petronis 1995; Grant and Dawson 1998; Kandel et al. 1992; Yamaguchi and Kandel 1984). Thus, adolescence represents a window of particular susceptibility to alcohol exposure and its long-term implications.

In adolescence, a striatal-mediated increase in motivational drive for reward over prefrontal cognitive control leads to poor behavioural self-control compared to adults (Dahl 2008; Yurgelun-Todd 2007). The neuroanatomical pathway of motivation is complex but involves information flow through cortical-striatal pathways that can stimulate motor and behavioural outputs (Kalivas et al. 1999; Kolomiets et al. 2001; Masterman and Cummings 1997; Woodward et al. 1999). This information flow relies on extensive modulation from secondary motivational circuitry components to incorporate salient emotional, sensory and mnemonic inputs (Pennartz et al. 1994; Groenewegen et al. 1999). Within these networks, a basic compartmentalization of striatal function has been established, with reward, motivation and premotor cognition ascribed to the ventral region and behavioural initiation and habit formation to the dorsal striatum (Everitt and Robbins 2013; Vollstadt-Klein et al. 2010). It is suggested that alcohol use could disrupt this process and lead to long-term maladaptation that underlies the increased propensity to develop substance use disorders (Badanich et al. 2007; Guerri and Pascual 2010; Maldonado-Devincci et al. 2010; Sahr et al. 2004). However, whilst it has been established that dorsal striatal dopaminergic transmission is integral to the transition from recreational to habitual drug intake, the long-term impact of adolescent alcohol exposure upon this region remains unclear. Furthermore, the impact of adolescent ethanol exposure on adult self-administration of drugs of abuse other than ethanol and on drug-induced effects on adult dopamine dynamics is unknown.

Our hypothesis is that episodic binge-like ethanol exposure, typically present in adolescence, induces long-term neurobiological and behavioural alterations in brain regions and systems implicated in development of substance use disorders. In a recent study from our laboratory, the impact of voluntary adolescent ethanol drinking on in vivo dopamine dynamics in adult rats with and without a single amphetamine challenge was investigated (Palm and Nylander 2014). The results confirmed age-dependent basal and amphetamineinduced evoked dopamine release in ethanol-naïve animals, and in ethanol-drinking animals, a lower basal (i.e. potassiuminduced) release was found, whereas the response to a single amphetamine challenge was unaffected (Palm and Nylander 2014). However, the animals were single housed during the voluntary drinking period, and it was of further interest to examine the pharmacological effects of ethanol on dopamine dynamics without the possible confounding factors of single housing (Meyer and Bardo 2015) and variations in the consumed amount of ethanol. Therefore, the specific aim in the present study was to investigate the long-term effects of episodic binge-like ethanol exposure (i.e. three consecutive days per week) using the same ethanol dose in all animals. Ethanol was administered orally in the same animal strain and sex as in the previous study, i.e. male non-alcohol preferring outbred Wistar rats. One group of animals was used to characterise adult basal and amphetamine-induced dopaminergic dynamics in the dorsal striatum by chronoamperometric recordings. A second group of animals was used to observe the influence of adolescent ethanol intake upon adult intravenous amphetamine self-administration behaviour. Subsequent chronoamperometric characterisation of these animals allowed group comparisons between amphetamine intake and dorsal striatal dopamine dynamics to be examined. Through these methods, we have demonstrated that bingelike adolescent ethanol exposure causes in vivo alterations in adult dorsal striatal dopamine dynamics as evident by altered response to amphetamine challenge.

\section{Materials and methods}

\section{Animals}

All animal experiments were performed under approval of the Uppsala Animal Ethical Committee and following the principles of the Guide for the Care and Use of Laboratory Animals and the guidelines of the Swedish Legislation on Animal Experimentation (Animal Welfare Act SFS1998:56) and the European Communities Council Directive (86/609/EEC).

Pregnant female Wistar rats (RccHan: WI, gestation day 16) were sourced from Harlan Laboratories B.V. (Horst, The Netherlands) and single housed under standard conditions $\left(22{ }^{\circ} \mathrm{C}, 50 \pm 10 \%\right.$ humidity, $12 \mathrm{~h}$ light-dark cycle commencing at 06:00, ad libitum access to pellet food and tap water, masking background noise). This is the least sensitive phase during pregnancy and was chosen to minimise the influence of stress related to transit. No signs of negative impact from transport were noticed during acclimatisation in the animal facility, and the delivery was normal in all females. The litters were cross-fostered and mixed so each litter contained four female and six males. Only male offspring were used in the continuation of the experiment. Upon weaning (post-natal day (PND) 21), animals were group housed under standard conditions as described above. Treatment groups were randomised, and adolescent animals (PND 28) were exposed to either ethanol or water. Upon completion of adolescent treatments (PND 59), two divergent protocols were followed, detailed schematically in Fig. 1. 


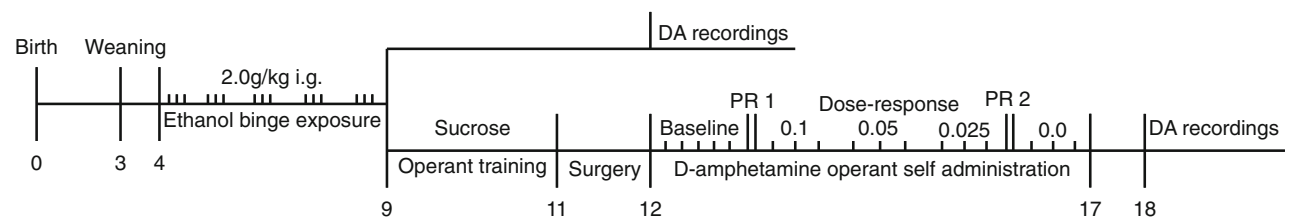

Fig. 1 Experimental outline showing the time points (weeks) for the behavioural and neurochemical analyses. Dopamine $(D A)$ recordings were done in vivo with high-speed chronoamperometry. $P R$ represents progressive ratio sessions with two different doses PR1 $(0.1 \mathrm{mg} / \mathrm{kg} / \mathrm{infu}-$ sion) and PR2 (0.05 mg/kg/infusion), $52 \times 14 \mathrm{~mm}(300 \times 300 \mathrm{DPI})$

\section{Drugs and solutions}

Ethanol, Solveco Etanol A 96 \% (Solveco AB, Rosersberg, Sverige), was diluted in tap water and D-amphetamine sulphate (Sigma-Aldrich, LLC, St. Louis, MO, USA) and diluted in sterile $\mathrm{NaCl} 9 \mathrm{mg} / \mathrm{mL}$ (Braun Melsungen AG, Melsungen, Germany). Sucrose food pellets (5-TUL, TestDiet, St. Louis, MO, USA) were used during the operant training. Anaesthesia agents were thiobutabarbital (Sigma-Aldrich, LSS, St. Louis, MO, USA), isoflurane (Forene Abbott, Solna, Sweden) or propofol (Braun Melsungen AG, Melsungen, Germany). For in vivo chronoamperometry, L-ascorbic acid, potassium chloride, sodium chloride, sodium phosphate and calcium chloride were purchased from Sigma-Aldrich, LLC (St. Louis, MO, USA) and diluted in Milli-Q water. For the post-operative care, buprenorphine (Schering-Plough, Brussels, Belgium), carpofen (Pfizer, Oy Animal Health, Helsinki, Finland) and amoxicillin (Ceva Animal Health, Dublin, Ireland) were used. For catheter maintenance, Heparin LEO (LEO Pharmaceuticals, Copenhagen, Denmark), sterile water (Braun Melsungen AG, Melsungen, Germany) and glycerol (Glycerol Unimedic AB, Matfors, Sweden) were used.

\section{Ethanol exposure}

The animals received intragastric administration of either water or ethanol $(2 \mathrm{~g} / \mathrm{kg}, 20 \% \mathrm{w} / \mathrm{v}$ ethanol diluted with water) for three consecutive days per week. This drinking paradigm was chosen to mimic common episodic adolescent drinking patterns and because intermittent ethanol exposure with drug-free days in-between has been shown to be necessary to induce neurobiological alterations similar to those seen in the transition to habitual and compulsive drinking (Spanagel 2003). The dose and route of administration were chosen to achieve binge-like oral consumption according to the National Institute on Alcohol Abuse and Alcoholism (NIAAA) definition of binge drinking $(>0.08 \mathrm{~g} / \mathrm{dl}$ in $2 \mathrm{~h}$ ) (NIAAA Drinking Levels Defined). Unpublished results from other experiments in our laboratory as well as in published data from others (Walker and Ehlers 2009) have shown that $2 \mathrm{~g} / \mathrm{kg}$ (intragastric) ethanol will produce blood alcohol concentrations reaching the binge criterion. Administrations were given at 09:00 on PND 28-30, 36-38, 43-45, 50-52, 57-59, followed by four days without treatment.

\section{In vivo dopamine recordings}

For animals that underwent ethanol exposure only (ethanol $n=9$, water $n=8$ ), dopamine recordings were taken between 11 and 12 weeks of age (corresponding to the age of the initiation of amphetamine self-administration for the second set of animals). For the animals that additionally underwent self-administration trials, dopamine recordings were conducted during weeks 18 and 19 (ethanol $n=5$, water $n=4$ ). In all cases, animals were drug-free for a minimum of 2 weeks before dopamine recordings commenced (Fig. 1).

Dopamine recordings were conducted using carbon fibre microelectrodes (SF1A, $30 \mu \mathrm{m}$ outer diameter, $150 \mu \mathrm{m}$ length, Quanteon, LLC, Nicholasville, KY, USA). A highspeed chronoamperometric protocol was utilised $(550 \mathrm{mV}$, $1 \mathrm{~Hz}$ sampling rate, $200 \mathrm{~ms}$ total) via a FAST16-mkII recording system (Quanteon). Electrode-pipette assemblies were prepared and calibrated immediately prior to in vivo recordings as previously described (Gerhardt and Hoffman 2001; Littrell et al. 2012). Briefly, electrodes were coated with Nafion (Sigma-Aldrich, LLC, St. Louis, MO, USA) and calibrated to cumulative additions of ascorbic acid and dopamine (ascorbic acid $250 \mu \mathrm{M}$, dopamine $2 \mu \mathrm{M}$ steps) applied to a bath of $0.05 \mathrm{M}$ phosphate-buffered saline. Electrodes used displayed a detection limit of $0.0237 \pm 0.0037 \mu \mathrm{M}$ and a selectivity of 3,864.35 \pm 881.16 for dopamine over ascorbic acid. Responses to dopamine were linear, with an average correlation coefficient $\left(R^{2}\right)$ of $0.827 \pm 0.025$ and an average reduction/ oxidation ratio of $0.628 \pm 0.011$ that is indicative of specific dopamine detection (Gerhardt and Hoffman 2001). After calibration, a micropipette filled with isotonic potassium chloride solution $\left(120 \mathrm{mM} \mathrm{KCl}, 29 \mathrm{mM} \mathrm{NaCl}, 2.5 \mathrm{mM} \mathrm{CaCl}_{2}\right.$, pH 7.27.4) was affixed with the tip 150-200 $\mu \mathrm{m}$ from the recording site of the electrode.

Animals were anaesthetized via intraperitoneal injection of $125 \mathrm{mg} / \mathrm{kg}$ thiobutabarbital and body temperature maintained with a thermostatic heating pad (Gaymar Industries, Inc., Orchard Park, New York). The electrode-pipette assembly was stereotaxically carefully placed in the dorsolateral striatum $(\mathrm{AP}+1.0, \mathrm{ML}+3.0, \mathrm{DV}-4.2 \mathrm{~mm})$ and an $\mathrm{Ag} / \mathrm{AgCl}$ reference electrode placed in the brain contralaterally and remote from working electrode-recording site.

After surgery and allowing $1 \mathrm{~h}$ for the stabilisation of electrode and surrounding tissue, $100 \mathrm{nl}$ of potassium chloride 
solution was locally ejected using pressure ejection (PicoSpritzer III, Parker Hannifin Corporation, Pine Brook, NJ, USA; ejection pressure $<22$ psi, ejection time $<2 \mathrm{~s}$ ), and resultant local dopamine release was detected by the electrode as a peak of rising dopamine concentration. Potassium chloride ejections were repeated every $10 \mathrm{~min}$ until three successive consistent dopamine releases were recorded for use as baseline reference peaks. Five minutes after the third reference peak was evoked, a single $2-\mathrm{mg} / \mathrm{kg}$ dose of amphetamine was injected via tail vein. Five minutes post-injection of amphetamine, dopamine release was evoked again, and this was then repeated every $10 \mathrm{~min}$ until $55 \mathrm{~min}$ post-drug. Upon termination of recording, animals were sacrificed, then the brain removed and frozen for subsequent histological identification of electrode location.

\section{Self-administration}

Rats from both the ethanol and water groups were subjected to assessment of self-administration behaviour followed by in vivo chronoamperometry (Fig. 1), the latter procedure is described above.

\section{Operant chamber apparatus}

Self-administration training and testing was conducted in sound-attenuated operant chambers (MED Associates Inc., Vermont, USA) equipped with two stimulus lights above two retractable stainless steel levers. A white house light placed on the wall opposite the levers was on during the entire session. A ventilating fan operated throughout the sessions and served as a masking noise. Intravenous solutions were delivered using an infusion pump (PHM-100, $3.33 \mathrm{rpm}$; Med Associates Inc.), and a 10-mL plastic syringe placed in the pump was connected to the implanted catheter through CoEx tubing (Harvard Apparatus, Kent, UK) and protected by a flexible metal leash (CamCaths, Ely, UK). Experiments were run and data collected by a PC with the MED-PC software (MedPC IV, MED Associates Inc., Vermont USA). The experiments were conducted in the same boxes for both training and test sessions, and ethanol- and water-treated animals were processed simultaneously throughout all phases of the self-administration procedure.

\section{Sucrose training}

Food restriction was initiated $48 \mathrm{~h}$ following the last intragastric pre-treatment session and was maintained throughout the self-administration training period to motivate food-seeking behaviour. Animal weights were carefully monitored and were not allowed to decrease more than $15 \%$ from commencement of food restriction. After 2 days on food restriction, the training to self-administer $45 \mathrm{mg}$ sucrose food pellets on a fixed ratio-1 (FR1) schedule was initiated. Each session started when the house light illuminated and the retractable levers were extended. A press on the active lever resulted in retraction of the lever and illumination of the stimulus light above the lever during a 10 -s time-out period. The criteria for fulfilled selfadministration training were accomplishment of 100 active lever presses within $30 \mathrm{~min}$ and a specificity $>0.85$ for the active lever.

\section{Surgery}

Intravenous catheters (CamCaths, Ely, UK) were implanted into the right jugular vein under isoflurane anaesthesia. Rats were administered post-operative analgesia (buprenorphine, $0.06 \mathrm{mg} / \mathrm{kg}$ s.c.; carpofen $5 \mathrm{mg} / \mathrm{kg}$ s.c.) and antibiotic (amoxicillin, $0.5 \mathrm{~mL} / \mathrm{kg}$ s.c.). Catheters were flushed with a heparin solution $(50 \mathrm{U} / \mathrm{mL})$ before and after every session, and a heparinized glycerol lock solution (50:50 heparin/glycerol) was used over weekends. Catheter patency was tested before the start and at the end of the study with an infusion of the short-acting anaesthetic agent propofol.

\section{Intravenous amphetamine self-administration}

After a minimum of 4 days of recovery from surgery, rats (ethanol $n=8$, water $n=8$ ) were allowed to self-administer amphetamine on a daily 60 min FR3 schedule of reinforcement. Each session started when the house light was turned on and the retractable levers were extended. Three responses on the active lever resulted in an intravenous infusion of amphetamine $(0.1 \mathrm{mg} / \mathrm{kg} /$ infusion $)$, and a 10 -s time-out period was initiated when both levers were retracted and a white stimulus light above the active lever was turned on. The lever designated to be the active lever was switched between sucrose training and intravenous self-administration. The maximum number of rewards during the 60 -min baseline sessions was set to 20. A press on the inactive lever had no programmed consequences but was recorded by the software.

The rats underwent five baseline sessions of $60 \mathrm{~min}$ FR3 amphetamine $(0.1 \mathrm{mg} / \mathrm{kg} /$ infusion $)$ before the operant requirements were switched to the progressive ratio (PR) format. Under this schedule of reinforcement, the response requirement started at 1 and escalated for each drug infusion delivered according to following scheme: 1, 2, 4, 6, 9, 12, 15, 20, $25,32,40,50,62,77,95,118,145,178,219,268,328,402$, 492, 603, 737 and 901 (see Richardson and Roberts 1996). The PR schedule was tested at two doses $(0.1$ and $0.05 \mathrm{mg} / \mathrm{kg}$ per infusion) for two consecutive days each (see Fig. 1). The sessions ended when $1 \mathrm{~h}$ had passed since the last reward or after a maximum session time of $4 \mathrm{~h}$. The breakpoint was 
defined as the total number of infusions during the session. Additionally, to test the dose-response function on a FR schedule, the unit dose of amphetamine $(0,0.025,0.05$ or $0.1 \mathrm{mg} / \mathrm{kg}$ per infusion) was varied, and each dose was tested for three consecutive 90 min FR3 sessions (Fig. 1). The first session of each dose in PR and dose-response trials was considered an acclimatisation session, and data collected during this session was not used in the statistical analysis.

\section{Data analysis}

\section{Dopamine analysis}

The main parameters examined from dopamine oxidation currents, i.e. the peak area, the maximal amplitude $(\mu \mathrm{M})$ of evoked peaks and the time taken for dopamine concentration to decline to $20 \%$ of the maximum for each peak, T80 (seconds), were analysed with the FAST analysis software (version 5.2; Quanteon, KY, USA). The amplitude is a measurement of dopamine release; T80 is the uptake measure, whereas the peak area encompasses both the dopamine release and reuptake of dopamine. The chronoamperometric recordings of amplitude and T80 allow analysis of both the release and reuptake inhibition action of amphetamine.

For comparison of reference peaks, the raw values of the above parameters for the first three consecutive consistent dopamine releases obtained were compared via a repeated measures ANOVA to examine the influence of time, adolescent treatment group and time-group effects (Statistica 10; StatSoft Inc., Tulsa, OK, USA).

For analysis of amphetamine challenge, mean baseline values of parameters were obtained from the three reference peaks for each animal and subsequent values described as a percentage of this baseline. Subsequently, repeated measures ANOVA were used to examine time, adolescent treatment group and time-group effects. Where time-group differences were observed $(p<0.05)$, the Tukey's HSD post hoc test was applied.

\section{Self-administration}

Student's $t$ test was used to compare the PR trials and sucrose pellet operant training. The parameters tested during operant training were the number of days until the rats had fulfilled the training criteria and the specificity for the active lever (number of presses on the active lever/total number of lever presses) when the criteria were fulfilled. The analysis of amphetamine self-administration behaviour on FR schedules was done with repeated measures ANOVA.

\section{Results}

\section{Dopamine recordings after adolescent ethanol exposure}

\section{Reference values}

Adolescent ethanol exposure alone did not affect evoked dopamine dynamics in adult animals in the basal state as evidenced by no statistically significant main effect of treatment across the three reference peaks (repeated measures ANOVA). The measurements (mean \pm SEM) in water controls and ethanol-exposed rats, respectively, were as follows: amplitude, $3.43 \pm 0.54$ and $2.62 \pm 0.39[F(1,15)=1.5, p=0.23]$; area, $42.0 \pm 8.87$ and $33.1 \pm 6.57[F(1,15)=0.66, p=0.43]$; and $\mathrm{T} 80$, $12.8 \pm 0.74$ and $13.4 \pm 1.18[F(1,15)=0.15, p=0.70]$. Further, there was no interaction effect between time and treatment; amplitude $[F(2,30)=0.46, p=0.63]$, area $[F(2,30)=0.18, p=$ $0.83]$ and $\mathrm{T} 80[F(2,30)=0.043, p=0.96]$.

\section{Amphetamine response after adolescent ethanol exposure}

Acute amphetamine administration (single dose, $2 \mathrm{mg} / \mathrm{kg}$, i.v.) resulted in significant increases in evoked dopamine peak area over time [effect of time: $F(8,120)=19.4, p<0.001$ ]. No main effect of adolescent ethanol exposure was found in the peak area [effect of treatment: $F(1,15)=2.30, p=0.15)]$. A significant interaction effect was observed indicating different amphetamine response in the two adolescent treatment groups [time $\times$ treatment: $F(8,120)=2.03, p=0.048$ ]. The post hoc analysis revealed that animals exposed to ethanol had a significant $(p=0.007)$ increased peak area $5 \mathrm{~min}$ after the amphetamine challenge, whereas the water-exposed animals had a prolonged increase in peak area with a statistically significant increase at $5(p<0.001), 15(p<0.001), 25(p=0.001)$ and 35 $(p=0.007)$ min after the amphetamine challenge.

Analysing the parameters included in the peak area revealed that the difference was mainly driven by an interaction effect in peak amplitude [time $\times$ treatment: $F(8,120)=1.93$, $p=0.061]$ (Fig. 2a). A main effect of time $[F(8,120=9.79$ $p<0.0001]$ was found, but no main effect of treatment alone was seen in the amplitude $[F(1,15)=1.63 p=0.22]$. In the T80 values, there were no main effect of treatment $[F(1,15)=0.40$ $p=0.53$ ] or interaction effect [time $\times$ treatment: $F(8,120)=$ $0.50, p=0.85]$ but a main effect of time $[F(8,120)=26.96$, $p<0.0001$ ] (Fig. 2b).

\section{Effects of adolescent ethanol exposure and adult operant self-administration}

\section{Sucrose training}

There was no between-group difference in the numbers of days until the rats had fulfilled their sucrose training (water 

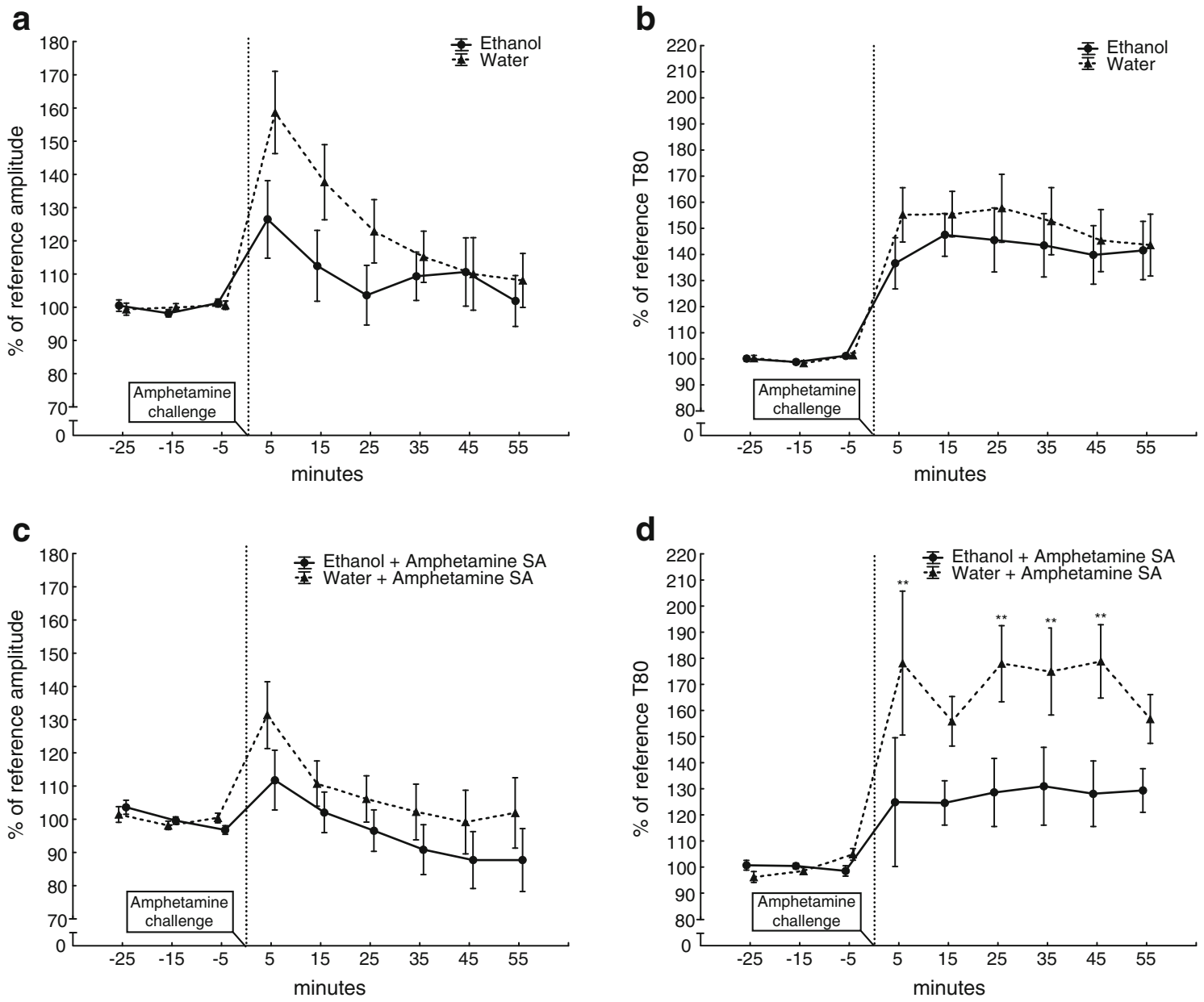

Fig. 2 Amplitudes (a and $\mathbf{c}$ ) and T80 (b and d) measurements over time after different drug exposures. The graphs show the responses after an intravenous amphetamine challenge $(2 \mathrm{mg} / \mathrm{kg}$ i.v.) as percent (mean \pm SEM) of reference values after adolescent ethanol $(n=9$, circles $)$ or water ( $n=8$, triangles) exposure (a and $\mathbf{b})$ and after adolescent ethanol

( $n=5$, circles $)$ or water $(n=4$, triangles) exposure followed by amphetamine self-administration (c and d). ${ }^{*} p<0.01$ compared to the time point $(-5)$ before the amphetamine challenge, repeated measures ANOVA followed by Tukey's HSD test, $150 \times 131 \mathrm{~mm}(300 \times 300 \mathrm{DPI})$

controls, $6.4 \pm 0.4$; ethanol-exposed rats, $6.2 \pm 0.4 ; t=-0.24$, $p=0.81$ ) or the specificity for the active lever (water controls, $0.94 \pm 0.02$; ethanol-exposed rats, $0.94 \pm 0.01 ; t=-0.11, p=$ 0.91 ) at the last day of the sucrose training.

\section{Amphetamine self-administration}

Repeated measures ANOVA revealed no main effect of treatment $[F(1,13)=1.65, p=0.22]$ or interaction effect between treatment and session $[(F(4,52)=0.29, p=0.88)]$ in the five baseline sessions (Fig. 3). No main effect of treatment ( $F(1$, $14)=1.06, p=0.32$ ) or interaction effect between dose and treatment $[F(3,42)=0.2, p=0.89]$ was found during the FR schedule dose-response function (Fig. 4). The rat motivational drive to consume amphetamine was tested in PR trials at two different doses and shown in Fig. 5; the breakpoint value did not differ between the two groups at any dose: $0.1 \mathrm{mg} / \mathrm{kg} /$ infusion trial $(t=0.52 ; p=0.61), 0.05 \mathrm{mg} / \mathrm{kg} /$ infusion trial $(t=$ $-0.070, p=0.94)$.

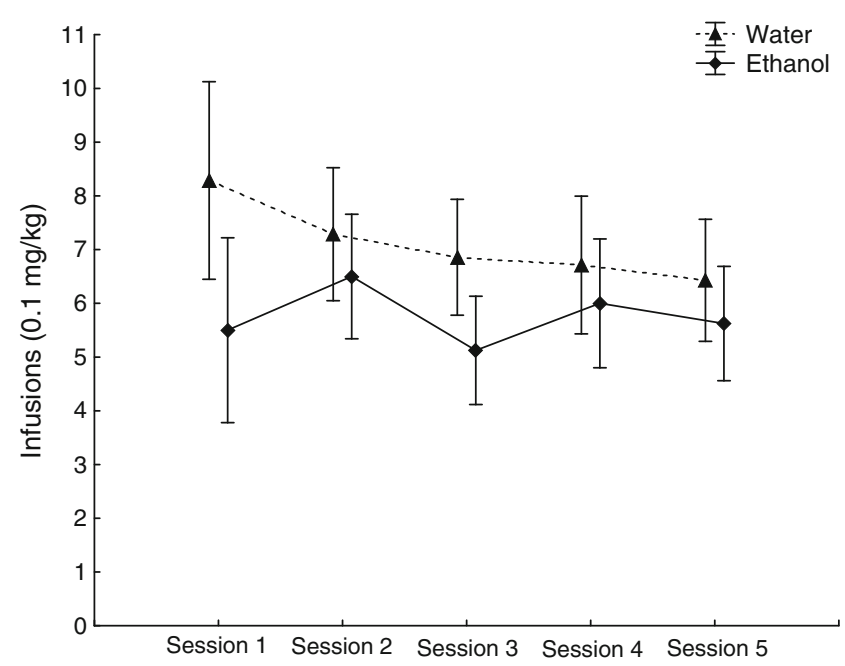

Fig. 3 Acquisition of amphetamine self-administration. The values represent mean $( \pm$ SEM) number of amphetamine infusions earned on a fixed ratio 3 schedule across five baseline sessions. Water $(n=8)$ and ethanol $(n=8), 90 \times 70 \mathrm{~mm}(300 \times 300 \mathrm{DPI})$ 


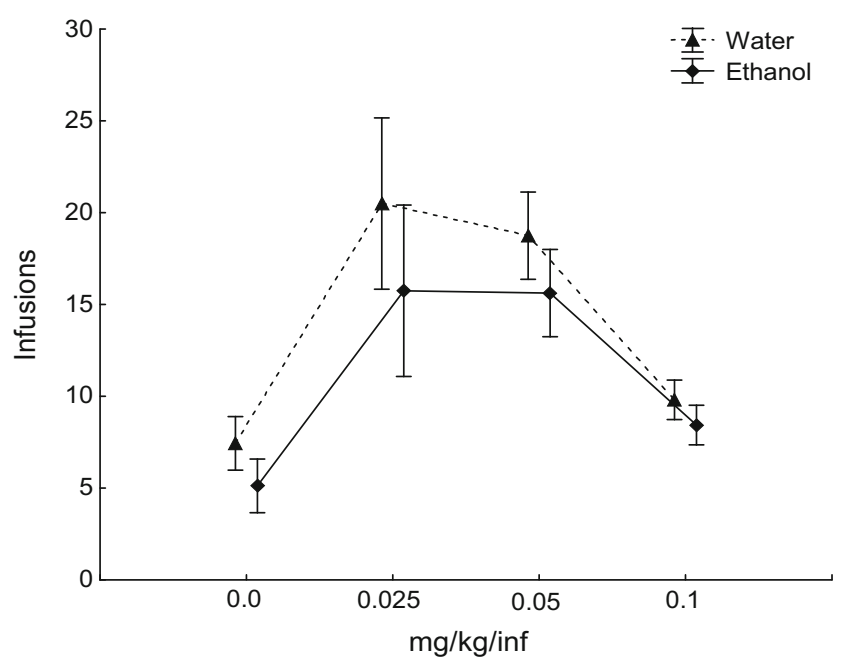

Fig. 4 Dose-response function for amphetamine self-administration. The values represent mean $( \pm \mathrm{SEM})$ number of amphetamine infusions earned on a fixed ratio 3 schedule across different unit doses. Water $(n=8)$ and ethanol $(n=8), 92 \times 71 \mathrm{~mm}(300 \times 300 \mathrm{DPI})$

\section{Challenge with amphetamine after adolescent ethanol exposure and adult amphetamine self-administration}

\section{Reference peaks}

No main effect of treatment was observed in adult reference peaks after amphetamine self-administration (repeated measures ANOVA). The measurements (mean \pm SEM) in water controls and ethanol-exposed rats, respectively, were as follows: amplitude, $2.86 \pm 0.27$ and $3.96 \pm 0.42[F(1,7)=1.25$, $p=0.30]$; area, $53.5 \pm 7.65$ and $76.1 \pm 9.29[F(1,7)=1.01, p=$

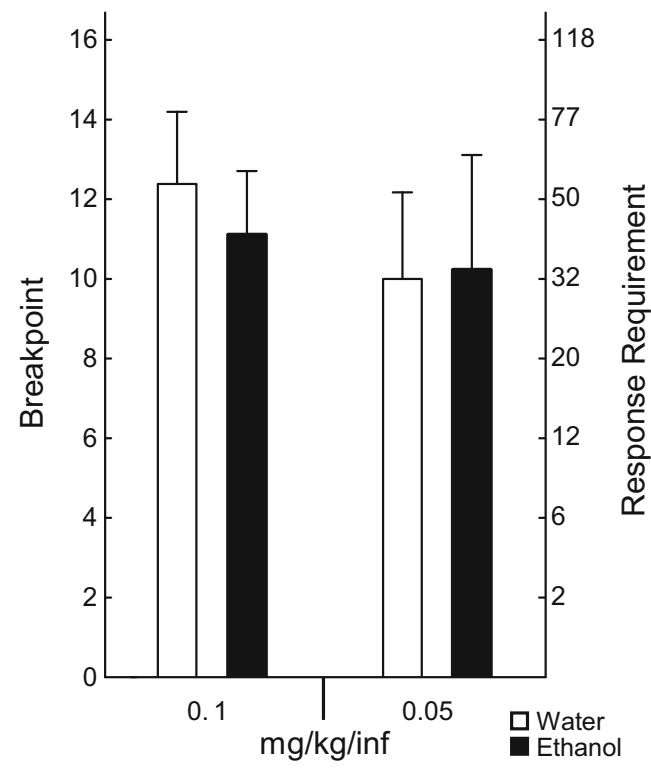

Fig. 5 Breakpoint values (rewards earned during the trial) and corresponding response requirements for the last earned reward when amphetamine was self-administered on a progressive ratio schedule of reinforcement. Water $(n=8)$ and ethanol $(n=8)$. Bars represent mean+ SEM, $83 \times 97 \mathrm{~mm}(300 \times 300 \mathrm{DPI})$
$0.34]$; and T80, $17.8 \pm 0.85$ and $18.1 \pm 0.44[F(1,7)=0.04, p=$ $0.85]$. Also, no interaction effect between time and treatment was observed: amplitude $[F(2,14)=1.1349, p=0.35]$, area $[F$ $(2,14)=1.97, p=0.18]$ and $\mathrm{T} 80[F(2,14)=3.20, p=0.07]$.

\section{Amphetamine response after adolescent ethanol exposure} and adult amphetamine self-administration

After self-administration, a single amphetamine challenge ( $2 \mathrm{mg} / \mathrm{kg}$, i.v.) resulted in significant increases in evoked dopamine peak area over time [effect of time: $F(8,56)=7.35$, $p<0.00001]$, and significant differences were observed in amphetamine response between the two adolescent treatment groups [effect of treatment: $F(1,7)=8.44, p=0.023$ ]. An interaction effect between time and treatment $[F(8,56)=3.56$, $p=0.0021]$ was found. The post hoc test showed that the ethanol-exposed rats did not increase their peak area after the amphetamine challenge, whereas water-exposed animals had an increased peak area $5(p<0.001), 15(p=0.02), 35$ ( $p=$ $0.05)$ and $45(p=0.006)$ min after the amphetamine challenge.

Further analysis of the parameters included in the peak area revealed that the difference in peak area was mainly driven by the T80 values where main effects of treatment $[F(1,7)=6.84$, $p=0.035]$ and time $[F(8,56)=10.67, p<0.0001]$ as well as an interaction effect between time and treatment $[F(8,56)=2.28$, $p=0.035]$ were found (Fig. $2 \mathrm{~d}$ ). The reduced T80 in ethanolexposed rats suggests a more efficient removal of dopamine. In the amplitude values, a main effect of time $[F(8,56)=5.69$, $p<0.0001]$ was found but no main effect of treatment $[F(1$, $7)=1.36, p=0.28]$ or interaction effect between time and treatment $[F(8,56)=1.01, p=0.44]$ (Fig. 2b).

\section{Discussion}

The present study is, to the best of our knowledge, the first to investigate both in vivo dopamine dynamics in dorsal striatum as well as amphetamine self-administration after adolescent binge-like ethanol exposure. The study provides new evidence for long-term alterations in dopamine dynamics within the dorsal striatum. Specifically, the consequences of being exposed to ethanol during adolescence were revealed when the animals were subjected to amphetamine challenge in adulthood.

\section{Adolescent ethanol exposure and adult in vivo dopamine}

The dopaminergic system in dorsal striatum undergoes significant changes during adolescence. Dopaminergic receptors (Gelbard et al. 1989; Giorgi et al. 1987; Teicher et al. 1995), tyrosine hydroxylase (Mathews et al. 2009; Matthews et al. 2013), dopamine transporters (Matthews et al. 2013; Truong et al. 2005) as well as the readily releasable and storage pool of 
dopamine (Stamford 1989) all differ between adolescents and adults. Therefore, the disruption of any aspect within this maturational process by repeated exposure of ethanol can be expected to impact dopamine function in adulthood.

The present data show that adolescent ethanol exposure had no effect on basal dopamine dynamics but resulted in reduced evoked peak area after amphetamine challenge in adult rats. Furthermore, the results indicate that suppressed dopamine release rather than more efficient dopamine removal contributed to this effect. Recent chronoamperometric studies conducted in our laboratory show that voluntary adolescent drinking significantly reduces basal (i.e. preamphetamine challenge) potassium-induced peak amplitudes in adulthood but did not affect dopamine responses to a single amphetamine challenge (Palm and Nylander 2014). The compiled results show that the impact of episodic adolescent ethanol exposure differs depending on ethanol intake paradigm. The discrepancies between the chronoamperometric results from the two studies can be a consequence of different study populations; the present study describes the effects of ethanol binges during adolescence independent of phenotype, whereas the results from Palm and Nylander (2014) describe ethanol effects in a drinking phenotype. The present study employed a forced administration regimen instead of voluntary drinking to be able to target the pharmacological effects of a pre-set ( $2 \mathrm{~g} / \mathrm{kg}$ i.g. $)$ dose of ethanol, independent of drinking patterns and intake. A limitation with the forced administration is stress induced by the gavage procedure. Stress activation affects the dopamine systems and could therefore be a potential confounding factor (Ungless et al. 2010). However, a recent study showed that intragastric administration had less impact on the HPA axis than intraperitoneal injections (Hoffman et al. 2014) which is in favour of the route used herein. On the other hand, voluntary drinking usually involves single housing which per se increases amphetamine self-administration in adulthood (Meyer and Bardo 2015) and would confound ethanol effects in the present experiment. Furthermore, many non-preferring rats do not ingest enough ethanol in a voluntary drinking model to achieve the BAC necessary enough for a "binge."

Other studies using microdialysis in mesolimbic regions have shown that repeated ethanol exposure increases basal extracellular levels of dopamine in adolescent rats (Badanich et al. 2007; Pascual et al. 2009; Philpot et al. 2009), but these effects did not last into adulthood (Pascual et al. 2009). Also in line with our findings of lower dopamine response after a drug challenge, intermittent ethanol exposure (i.p.) during adolescence decreased accumbal ethanol-evoked dopamine release 7 and 14 days after treatment, but the effect was diminished after 28 days (Zandy et al. 2014). Furthermore, repeated treatment with ethanol in preadolescence (PND 21-24) or early adolescence (PND 31-34) lowered dopamine efflux in nucleus accumbens after ethanol challenge (Philpot et al. 2009).

\section{Adolescent ethanol exposure and adult amphetamine self-administration}

It has previously been shown that a history of adolescent ethanol exposure increases subsequent voluntary intake of ethanol (Alaux-Cantin et al. 2013; Criado and Ehlers 2013; Pascual et al. 2009). However, the literature is not conclusive, several studies report subtle or no effect on subsequent intake (Criado and Ehlers 2013; Gilpin et al. 2012; Siegmund et al. 2005; Slawecki and Betancourt 2002; Vetter et al. 2007). Surprisingly, little work has investigated how adolescent ethanol exposure influences intake of drugs of abuse other than ethanol. With regard to the growing evidence that adolescent ethanol exposure affects the dopamine transmission, drugs that specifically target this system are of interest. It has been shown that ethanol exposure during early/mid-adolescence (PND 30-39) sensitised the rewarding effects of cocaine and attenuated the aversive effects as well as altered gross locomotor activity (Hutchinson and Riley 2012; Hutchison et al. 2010). The impact on amphetamine-induced reward is not known, and since amphetamine was used as a challenge drug in the present study, it was of interest to further assess the initial rewarding effects of amphetamine in the selfadministration paradigm.

Since cognitive and behavioural dysfunction can be seen after adolescent ethanol exposure (for review, see Guerri and Pascual 2010), sucrose training was performed before initiating the amphetamine self-administration. No differences between the groups were found in any of the parameters tested during the sucrose training, indicating that the ethanol exposure had no effect on the learning ability for the operant procedure and did not affect the general motivation for a non-drug reward.

Analysis of the self-administration behaviour revealed that there were no differences between the groups in their initial response to amphetamine (i.e. baseline sessions). With the concept of a dorsal striatal involvement in the shift to habitual drug use (Everitt and Robbins 2013) in mind, we anticipated that there might be a difference between the groups motivational drive to consume amphetamine. However, no differences between water- and ethanol-exposed rats were found in the PR trials (i.e. 0.1 or $0.05 \mathrm{mg} / \mathrm{kg}$ per infusion). A dose-response function was used to further explore the selfadministration behaviour on the FR schedule, but the groups displayed similar intake behaviour in all doses tested.

Our hypothesis, based on previous literature and the findings from our own experiments, was that dorsal striatal dopamine and amphetamine self-administration would be affected by adolescent ethanol exposure. The noted difference in peak area, driven by reduced release of dopamine (reduced amplitude) after amphetamine challenge in the ethanol-exposed group, had, however, no influence on drug-taking behaviour during the short period of self-administration used herein to 
examine initial drug reward. Nevertheless, there are a number of ways to modify the amphetamine self-administration paradigm, and our results might not be conclusive. A different setup with prolonged self-administration or a shift toward lower doses might reveal more subtle alterations between the groups. Amphetamine is a potent drug, and the doses used and the length of the exposure periods might overrule the effects of adolescent ethanol exposure. To observe specific subgroups in relation to adolescent influence upon drug intake and responding, specific inclusion criteria (e.g. a certain number of rewards per session) are commonly used. In the present study however, all animals displaying amphetamine selfadministration behaviour were included, including the low responding animals. The ethanol exposure during adolescence was independent of phenotype, and we wanted to keep this heterogeneity throughout the experiment. Importantly, all animals included were familiarised with the operant technique (i.e. the sucrose training) before the initiation of amphetamine self-administration. In both the fixed and progressive ratio trials, large individual differences in amphetamine intake were also found that were independent of adolescent ethanol exposure.

\section{Adolescent ethanol exposure, amphetamine self-administration and in vivo dopamine}

In rats, with or without a history of adolescent ethanol exposure, in vivo dopamine dynamics were analysed in the dorsal striatum 2 weeks after the last amphetamine selfadministration session. The response to a single challenge with amphetamine was attenuated in animals with both adolescent ethanol exposure and repeated adult amphetamine self-administration as compared to those with only adult amphetamine intake. The reduced response was driven by an abolished effect on T80 (the time for removal) possibly indicating different effects on transporter function. Thus, even though no differences were noted in amphetamine intake in the self-administration assessment, the response to the drug in the brain was altered in ethanol-exposed animals. These results indicate potential for a synergistic effect upon dopaminergic response to amphetamine after adolescent ethanol and adult amphetamine exposure. An interesting aspect would have been to investigate how these rats responded to a reintroduction of amphetamine self-administration, for example another PR trial, lower unit doses of amphetamine or extended repeated periods of self-administration.

To summarise our findings, male rats exposed to ethanol in adolescence had, as adults, somewhat reduced dopamine release after a single amphetamine challenge but no differences in amphetamine self-administration with the current experimental setup. However, amphetamine challenge after adult amphetamine intake through self-administration revealed pronounced differences in striatal dopamine removal between animals with or without a history of adolescent ethanol exposure. Such difference in drug-induced dopamine response in ethanol-exposed individuals may affect their further drugtaking behaviour. If so, it can be speculated that a vulnerability for later excessive drug administration previously reported after adolescent ethanol exposure may not be seen at the first contacts with a drug in young adults but rather emerge with repeated drug use. However, this was not tested in the present study, and further studies are warranted to elucidate the relevance of the altered drug response reported herein for altered drug taking as a consequence of adolescent ethanol exposure as well as investigate possible differences between the sexes.

Acknowledgments Financial support was gratefully received from the Alcohol Research Council of the Swedish Alcohol Retailing Monopoly, ERAB: the European Foundation for Alcohol Research (EA 11 30), and the Swedish Research Council (K2012-61X-22090-01-3).

Conflict of interest The authors declare that they have no competing interests.

Open Access This article is distributed under the terms of the Creative Commons Attribution 4.0 International License (http://creativecommons.org/licenses/by/4.0/), which permits unrestricted use, distribution, and reproduction in any medium, provided you give appropriate credit to the original author(s) and the source, provide a link to the Creative Commons license, and indicate if changes were made.

\section{References}

Adriani W, Laviola G (2003) Elevated levels of impulsivity and reduced place conditioning with d-amphetamine: two behavioral features of adolescence in mice. Behav Neurosci 117:695-703

Alaux-Cantin S, Warnault V, Legastelois R, Botia B, Pierrefiche O, Vilpoux C, Naassila M (2013) Alcohol intoxications during adolescence increase motivation for alcohol in adult rats and induce neuroadaptations in the nucleus accumbens. Neuropharmacology 67:521-31. doi:10.1016/j.neuropharm.2012.12.007

Anthony JC, Petronis KR (1995) Early-onset drug use and risk of later drug problems. Drug Alcohol Depend 40:9-15

Arnett J (1992) Reckless behaviour in adolescence: a developmental perspective. Dev Rev 12:339-373

Badanich KA, Maldonado AM, Kirstein CL (2007) Chronic ethanol exposure during adolescence increases basal dopamine in the nucleus accumbens septi during adulthood. Alcohol Clin Exp Res 31:895900. doi:10.1111/j.1530-0277.2007.00370.x

Criado JR, Ehlers CL (2013) Effects of adolescent onset voluntary drinking followed by ethanol vapor exposure on subsequent ethanol consumption during protracted withdrawal in adult Wistar rats. Pharmacol Biochem Behav 103:622-30. doi:10.1016/j.pbb.2012. 10.016

Dahl RE (2008) Biological, developmental, and neurobehavioral factors relevant to adolescent driving risks. Am J Prev Med 35:S278-84. doi:10.1016/j.amepre.2008.06.013

Dawson DA, Goldstein RB, Chou SP, Ruan WJ, Grant BF (2008) Age at first drink and the first incidence of adult-onset DSM-IV alcohol use disorders. Alcohol Clin Exp Res 32:2149-2160. doi:10.1111/J. 1530-0277.2008.00806.X 
DeWit DJ, Adlaf EM, Offord DR, Ogborne AC (2000) Age at first alcohol use: a risk factor for the development of alcohol disorders. Am J Psychiatry 157:745-50

Everitt BJ, Robbins TW (2013) From the ventral to the dorsal striatum: devolving views of their roles in drug addiction. Neurosci Biobehav Rev 37:1946-1954. doi:10.1016/J.Neubiorev.2013.02.010

Gelbard HA, Teicher MH, Faedda G, Baldessarini RJ (1989) Postnatal development of dopamine D1 and D2 receptor sites in rat striatum. Brain Res Dev Brain Res 49:123-30

Gerhardt GA, Hoffman AF (2001) Effects of recording media composition on the responses of Nafion-coated carbon fiber microelectrodes measured using high-speed chronoamperometry. J Neurosci Methods 109:13-21

Gilpin NW, Karanikas CA, Richardson HN (2012) Adolescent binge drinking leads to changes in alcohol drinking, anxiety, and amygdalar corticotropin releasing factor cells in adulthood in male rats. PLoS One 7, e31466. doi:10.1371/journal.pone.0031466

Giorgi O, De Montis G, Porceddu ML, Mele S, Calderini G, Toffano G, Biggio G (1987) Developmental and age-related changes in D1dopamine receptors and dopamine content in the rat striatum. Brain Res 432:283-90

Grant BF, Dawson DA (1997) Age at onset of alcohol use and its association with DSM-IV alcohol abuse and dependence: results from the National Longitudinal Alcohol Epidemiologic Survey. J Subst Abus 9:103-110. doi:10.1016/S0899-3289(97)90009-2

Grant BF, Dawson DA (1998) Age of onset of drug use and its association with DSM-IV drug abuse and dependence: results from the National Longitudinal Alcohol Epidemiologic Survey. J Subst Abus 10:16373

Groenewegen HJ, Wright CI, Beijer AV, Voorn P (1999) Convergence and segregation of ventral striatal inputs and outputs. Ann N Y Acad Sci 877:49-63

Guerri C, Pascual M (2010) Mechanisms involved in the neurotoxic, cognitive, and neurobehavioral effects of alcohol consumption during adolescence. Alcohol 44:15-26. doi:10.1016/j.alcohol.2009.10. 003

Hawkins JD, Graham JW, Maguin E, Abbott R, Hill KG, Catalano RF (1997) Exploring the effects of age of alcohol use initiation and psychosocial risk factors on subsequent alcohol misuse. J Stud Alcohol 58:280-290

Hoffman J, Philpot, R., Kirstein C. (2014) Comparison of stress response to oral gavage and intraperitoneal injection Research Society on Alcoholism. Alcoholism: Clinical and Experimental Research, Bellevue, Washington, pp 1A-292A

Hutchinson MA, Riley AL (2012) Ethanol exposure during either adolescence or adulthood alters the rewarding effects of cocaine in adult rats. Pharmacol Biochem Behav 101:458-464. doi:10.1016/j.pbb. 2012.02.007

Hutchison MA, Albaugh DL, Riley AL (2010) Exposure to lcohol during adolescence alters the aversive and locomotor-activating effects of cocaine in adult rats. Pharmacol Biochem Behav 97:370-376. doi: 10.1016/j.pbb.2010.09.006

Kalivas PW, Churchill L, Romanides A (1999) Involvement of the pallidal-thalamocortical circuit in adaptive behavior. Ann N Y Acad Sci 877:64-70. doi:10.1111/j.1749-6632.1999.tb09261.x

Kandel DB, Yamaguchi K, Chen K (1992) Stages of progression in drug involvement from adolescence to adulthood: further evidence for the gateway theory. J Stud Alcohol 53:447-57

Kolomiets BP, Deniau JM, Mailly P, Ménétrey A, Glowinski J, Thierry AM (2001) Segregation and convergence of information flow through the cortico-subthalamic pathways. J Neurosci Off J Soc Neurosci 21:5764-5772

Littrell OM, Pomerleau F, Huettl P, Surgener S, McGinty JF, Middaugh LD, Granholm AC, Gerhardt GA, Boger HA (2012) Enhanced dopamine transporter activity in middle-aged Gdnf heterozygous mice.
Neurobiol Aging 33(427):e1-14. doi:10.1016/j.neurobiolaging. 2010.10 .013

Maldonado-Devincci AM, Badanich KA, Kirstein CL (2010) Alcohol during adolescence selectively alters immediate and long-term behavior and neurochemistry. Alcohol 44:57-66. doi:10.1016/j. alcohol.2009.09.035

Masterman DL, Cummings JL (1997) Frontal-subcortical circuits: the anatomic basis of executive, social and motivated behaviors. J Psychopharmacol Oxf Engl 11:107-114

Mathews IZ, Waters P, McCormick CM (2009) Changes in hyporesponsiveness to acute amphetamine and age differences in tyrosine hydroxylase immunoreactivity in the brain over adolescence in male and female rats. Dev Psychobiol 51:417-28. doi:10.1002/dev.20381

Matthews M, Bondi C, Torres G, Moghaddam B (2013) Reduced presynaptic dopamine activity in adolescent dorsal striatum. Neuropsychopharmacology 38:1344-51. doi:10.1038/npp.2013.32

Meyer AC, Bardo MT (2015) Amphetamine self-administration and dopamine function: assessment of gene $\mathrm{x}$ environment interactions in Lewis and Fischer 344 rats. Psychopharmacol (Berl). doi:10.1007/ s00213-014-3854-1

Nasrallah NA, Clark JJ, Collins AL, Akers CA, Phillips PE, Bernstein IL (2011) Risk preference following adolescent alcohol use is associated with corrupted encoding of costs but not rewards by mesolimbic dopamine. Proc Natl Acad Sci U S A 108:5466-71. doi:10.1073/pnas. 1017732108

Nasrallah NA, Yang TW, Bernstein IL (2009) Long-term risk preference and suboptimal decision making following adolescent alcohol use. Proc Natl Acad Sci U S A 106:17600-4. doi:10.1073/pnas. 0906629106

NIAAA Drinking Levels Defined. In: NIAAA (ed). National Institute on Alcohol Abuse and Alcoholism, http://www.niaaa.nih.gov/alcoholhealth/overview-alcohol-consumption/moderate-binge-drinking

Palm S, Nylander I (2014) Dopamine release dynamics change during adolescence and after voluntary alcohol intake. PLoS One 9, e96337. doi:10.1371/journal.pone.0096337

Pascual M, Boix J, Felipo V, Guerri C (2009) Repeated alcohol administration during adolescence causes changes in the mesolimbic dopaminergic and glutamatergic systems and promotes alcohol intake in the adult rat. J Neurochem 108:920-31. doi:10.1111/j.1471-4159. 2008.05835.x

Pennartz CMA, Groenewegen HJ, Lopes da Silva FH (1994) The nucleus accumbens as a complex of functionally distinct neuronal ensembles: An integration of behavioural, electrophysiological and anatomical data. Prog Neurobiol 42:719-761. doi:10.1016/03010082(94)90025-6

Philpot RM, Wecker L, Kirstein CL (2009) Repeated ethanol exposure during adolescence alters the developmental trajectory of dopaminergic output from the nucleus accumbens septi. Int J Dev Neurosci 27:805-15. doi:10.1016/j.ijdevneu.2009.08.009

Richardson NR, Roberts DC (1996) Progressive ratio schedules in drug self-administration studies in rats: a method to evaluate reinforcing efficacy. J Neurosci Methods 66:1-11

Sahr AE, Thielen RJ, Lumeng L, Li TK, McBride WJ (2004) Longlasting alterations of the mesolimbic dopamine system after periadolescent ethanol drinking by alcohol-preferring rats. Alcohol Clin Exp Res 28:702-11

Siegmund S, Vengeliene V, Singer MV, Spanagel R (2005) Influence of age at drinking onset on long-term ethanol self-administration with deprivation and stress phases. Alcohol Clin Exp Res 29: $1139-45$

Slawecki CJ, Betancourt M (2002) Effects of adolescent ethanol exposure on ethanol consumption in adult rats. Alcohol 26:23-30

Spanagel R (2003) Alcohol addiction research: from animal models to clinics. Best Pract Res Clin Gastroenterol 17:507-18

Spear LP (2000) The adolescent brain and age-related behavioral manifestations. Neurosci Biobehav Rev 24:417-63 
Stamford JA (1989) Development and ageing of the rat nigrostriatal dopamine system studied with fast cyclic voltammetry. J Neurochem 52:1582-9

Steinberg L (2008) A social neuroscience perspective on adolescent risktaking. Dev Rev 28:78-106. doi:10.1016/j.dr.2007.08.002

Steinberg L, Graham S, O'Brien L, Woolard J, Cauffman E, Banich M (2009) Age differences in future orientation and delay discounting. Child Dev 80:28-44. doi:10.1111/j.1467-8624. 2008.01244.x

Swendsen J, Burstein M, Case B, Conway KP, Dierker L, He J, Merikangas KR (2012) Use and abuse of alcohol and illicit drugs in US adolescents: results of the National Comorbidity SurveyAdolescent Supplement. Arch Gen Psychiatry 69:390-8. doi:10. 1001/archgenpsychiatry.2011.1503

Teicher MH, Andersen SL, Hostetter JC Jr (1995) Evidence for dopamine receptor pruning between adolescence and adulthood in striatum but not nucleus accumbens. Brain Res Dev Brain Res 89:167-72

Truong JG, Wilkins DG, Baudys J, Crouch DJ, Johnson-Davis KL, Gibb JW, Hanson GR, Fleckenstein AE (2005) Age-dependent methamphetamine-induced alterations in vesicular monoamine transporter-2 function: implications for neurotoxicity. J Pharmacol Exp Ther 314: 1087-92. doi:10.1124/jpet.105.085951

Ungless MA, Argilli E, Bonci A (2010) Effects of stress and aversion on dopamine neurons: implications for addiction. Neurosci Biobehav Rev 35:151-6. doi:10.1016/j.neubiorev.2010.04.006
Vetter CS, Doremus-Fitzwater TL, Spear LP (2007) Time course of elevated ethanol intake in adolescent relative to adult rats under continuous, voluntary-access conditions. Alcohol Clin Exp Res 31: 1159-68. doi:10.1111/j.1530-0277.2007.00417.x

Vollstadt-Klein S, Wichert S, Rabinstein J, Buhler M, Klein O, Ende G, Hermann D, Mann K (2010) Initial, habitual and compulsive alcohol use is characterized by a shift of cue processing from ventral to dorsal striatum. Addiction 105:1741-9. doi:10.1111/j.1360-0443. 2010.03022.x

Walker BM, Ehlers CL (2009) Age-related differences in the blood alcohol levels of Wistar rats. Pharmacol Biochem Behav 91:560-5. doi: 10.1016/j.pbb.2008.09.017

Woodward DJ, Chang JY, Janak P, Azarov A, Anstrom K (1999) Mesolimbic neuronal activity across behavioral states. Ann N Y Acad Sc. 877:91-112

Yamaguchi K, Kandel DB (1984) Patterns of drug use from adolescence to young adulthood: III. Predictors of progression. Am J Public Health 74:673-81

Yurgelun-Todd D (2007) Emotional and cognitive changes during adolescence. Curr Opin Neurobiol 17:251-7. doi:10.1016/j.conb.2007.03.009

Zandy SL, Matthews DB, Tokunaga S, Miller AD, Blaha CD, Mittleman $G$ (2014) Reduced dopamine release in the nucleus accumbens core of adult rats following adolescent binge alcohol exposure: age and dose-dependent analysis. Psychopharmacol (Berl). doi:10.1007/ s00213-014-3712-1 